\title{
Wayfinding From Multiple Sources of Local Information in Retinal Flow
}

\author{
James E. Cutting \\ Cornell University
}

\begin{abstract}
Although they concluded differently, N. G. Kim, R. Growney, and M. T. Turvey (1996) provided data that supports previous empirical (J. E. Cutting, K. Springer, P. A. Braren, \& S. H. Johnson, 1992) and metatheoretical (J. E. Cutting, 1986) claims. Their data show that, in simulations of pursuit fixation during locomotion through a cluttered environment, multiple sources of local information in retinal flow-previously analyzed as differential parallactic displacements (DMP), inward motion (IM), and outward deceleration (OD)-appear to be used by observers to determine the direction of their heading. Four alternative versions of parallax information were considered. Three were global sources-differential motion, spatial pooling, size-weighted spatial pooling; the other was a local source-the displacement direction of the largest object (DDLO) in the field. Of these, DDLO was best predictor, outstripping DMP and the 3 global sources; but IM and OD remained important. Thus, it remains that several local sources of motion information best predict the wayfinding data.
\end{abstract}

Over the past decade my colleagues and I have been developing a theory of how people find their way through cluttered environments with ease and without injury. We call this overall task wayfinding. In many cases it divides into several subtasks. First, one must often plot a course through an environment, taking into account local conditions of the terrain, such as puddles, walls, and sidewalks. Second, on the basis of visual, and perhaps vestibular and kinesthetic, information one must periodically check one's success in following that course. Third, on the basis of visual information one must avoid stationary and moving obstacles. And finally, if one is to return along an unfamiliar path, one must recognize landmarks from the reverse side, and guide oneself home accordingly.

Most psychological research has focused on an aspect of the second subtask-monitoring visual information to determine one's instantaneous heading, or aimpoint (e.g., Crowell \& Banks, 1993; Cutting, 1986; Cutting, Springer, Braren, \& Johnson, 1992; Van den Berg, 1992; Vishton \& Cutting, 1995; Warren \& Hannon, 1990; Warren \& Kurtz, 1992; Warren, Morris, \& Kalish, 1988). Others have considered the role of feedback from eye movements (Roy-

This research and analysis were supported by National Science Foundation Grant SBR-9212786.

I thank Nam-Gyoon Kim for sharing his data and, more important, for the analyses presented here, the coordinates for 7,072 trees associated with them; I thank William Warren for his criticisms and suggestions that prompted the new analyses here; I thank Peter Vishton for many things but particularly the discussion of the subtasks of wayfinding; and I thank Nan Karwan for her continued support.

Correspondence concerning this article should be addressed to James E. Cutting, Department of Psychology, Cornell University, 270 Uris Hall, Ithaca, New York 14853-7601. Electronic mail may be sent via Internet to jec7@comell.edu. den, Banks, \& Crowell, 1992), although their role is not always clear (Cutting, Vishton, Flückiger, Baumberger, \& Gerndt, in press; Van den Berg \& Brenner, 1994a, 1994b); others have considered the role of vestibular information (Berthoz, Israël, Georges-François, Grasso, \& Tsuzuku, 1995); and still others have begun to focus on the third task, avoiding collisions with stationary and moving objects (Cutting, Vishton, \& Braren, 1995; Warren \& Saunders, 1995).

With the bulk of the literature, however, I will focus here on an aspect of the second subtask, the ability of observers to determine the direction of their heading with respect to their gaze. Many different theoretical schemes have been promoted for how human beings might determine heading. These divide at least three ways-researchers have been concerned with single versus multiple sources of information, with retinal versus optical flow, and with global versus local information analyses. Consider each of these in turn.

First, with a concentration on invariants, researchers most sympathetic to the ecological approach to perception (e.g., Burton \& Turvey, 1990; Gibson, 1979) have tended to focus on single sources of information as they might constrain perception. Others (e.g., Cutting, 1986; Gilden \& Proffitt, 1989; Maloney \& Landy, 1989; Massaro, 1987) have focused on multiple sources of information for perception. This split can also be found in the wayfinding literature: Traditional approaches to wayfinding have typically focused on a single analysis of motion information (Gibson, 1966; Gibson, Olum, \& Rosenblatt, 1955), often deriving what has been called the focus of expansion, or radial flow pattern, in the optical array generated by the linear translation of an observer. In contrast, my approach has focused on multiple motion sources, which will be outlined below. ${ }^{1}$ 
Second, in the approach to wayfinding promoted by Gibson (1966) and his followers (e.g., Kim, Growney, \& Turvey, 1996), the radial flow pattern of optical flow can only become useful after the complex of flow information at the eye is decomposed into its components. That relation can be given by:

$$
\begin{aligned}
\text { retinal flow } & =\text { rotational flow } \\
& + \text { translational (optical) flow, }
\end{aligned}
$$

where retinal flow is the motion presented to the eye, rotational flow is that generated by pursuit fixation of the observer on a stationary object off the heading vector, and translational flow is that generated by the moving observer and containing the focus of expansion. ${ }^{2}$ All contemporary researchers appear to agree that the combined translational and rotational flow is the proximal stimulus during human movement; disagreement occurs over whether this pattern must be decomposed into its components, subtracting the rotational flow from retinal flow. If optical flow could be used to determine one's heading during linear movement, then the procedure by which aimpoint is determined seems straightforward: After decomposition there is a prominent radial motion pattern away from the point toward which one is headed. Methods proposed for such decomposition abound (see Warren, 1995; Warren et al., 1988 , for reviews), but I have suggested these are neither necessary nor typically used by human observers at pedestrian speeds (Cutting et al., 1992). How information might be used directly from retinal flow will also be discussed below.

Third, in analyzing the complex of motions generated by a moving observer, most researchers have assumed that global processes are at work. That is, to solve the wayfinding task the visual system may combine the motion information in various ways over reasonably large portions of the visual field. This application has made a certain amount of sense because most methodological approaches to wayfinding have used dot fields with many elements. In contrast, however, my approach has focused more locally on the motion of discrete elements in the visual field. This, too, has made a certain amount of sense because my displays have always used larger objects, such as schematic trees in a relatively sparse forest. At the end of this Observation I will compare the efficacy of several local and global approaches.

In the three sections that follow I will focus, in turn, on each of these different approaches to wayfinding--first on the multiple sources of motion information that I have isolated in the past, second on the manner in which they appear in retinal flow, and third on comparisons of local and global sources. In preview, the analyses presented support the title of this Observation and necessitate a revision in the conclusions of Cutting et al. (1992), replacing one local source of information with another.

\section{Multiple Sources of Local Information in Retinal Flow}

\section{Differential Parallactic Displacements}

Cutting et al. (1992) outlined several possible sources of information for wayfinding. The first is called differential parallactic displacements (DMP). ${ }^{3}$ When moving through a rigid environment on either a straight or curved path and when looking off to the side at a stationary object, a general heuristic can be applied to the motions as projected on the retina. This rule is

$$
N>-F,
$$

where $N$ stands for the motion of objects nearer than fixation (and given positive sign) and $F$ stands for the motion of objects farther away (see Cutting et al., 1992, for the derivation). If the environment is sufficiently cluttered and the observer is looking sufficiently far away, then the fastest moving object or texture in the visual field will be in the foreground. Thus, if this object is moving left, one's direction of movement is likely to be to the right of fixation; if it is moving right, heading is likely to the left. In terms of Equation 1, then, DMP is typically correlated with the direction of rotational field, and hence is opposite to the nominal direction of heading. Cutting (1986; Cutting et al., 1992) found that DMP accounted for most correct responses in a wayfinding task; Cutting et al. determined that its absence accounted for many errors as well.

DMP, however, is only one source isolated by Cutting et al. (1992). Following the tenets of directed perception (Cutting, 1986, 1991a, 1991b), in which more than one source may suffice to determine what should be perceived, Cutting et al. searched for others and found at least one, which we now call inward displacements (IM). That is, during pursuit

\footnotetext{
${ }^{1}$ Recently it has become apparent that still other information is pertinent to wayfinding. In addition to motion information, for example, static information has been shown to aid performance (Cutting et al., 1992, Experiment 2; Cutting et al., in press; Van den Berg \& Brenner, 1994a, 1994b; Vishton \& Cutting, 1995; Vishton, Nijhawan, \& Cutting, 1994). This static informationheight in the visual field, occlusion, relative size, and so forth-appears to serve as a support structure indicating relative depth and layout. Because heading is generated only by motion, however, the primary information can only be revealed through motion.

${ }^{2}$ Most researchers do not distinguish between retinal and optical flow, but consider optical flow sometimes to have a rotational component and sometimes not. The distinction, however, is Gibson's (1966) and was adopted by Cutting et al. (1992) and Kim et al. (1996).

3 The major information for wayfinding discussed here used to be called differential motion parallax (DMP); a second source used to be called inward motion (IM). Vishton and Cutting (1995), however, discovered that it is not the motion of objects in the retinal field that is important, but their displacements. Thus, we have renamed the information differential parallactic displacements and inward displacements. Because Kim et al. (1996) used the abbreviations of the older nomenclature, for purposes of clarity I do here as well.
} 
gaze off the side of one's path, some objects move toward the fovea and, in the two-dimensional projection of the scene, the fixated object. Such displacements can occur for objects both nearer and farther than fixation. Again, in terms of Equation 1, IM is correlated with the direction of eye or head rotation and can be used to detect the direction of heading. Cutting et al. found that the presence of IM accounted for many correct responses when DMP failed. Moreover, performance was near or below chance when neither source was present.

\section{Inward Displacements and Kindred Motions}

Because they play a central role in this article, and because they have been previously represented inappropriately (Cutting et al., 1992, Figure 13), IM and related motions will be considered here in more detail. ${ }^{4}$ As shown in Figure 1 there are three such motions for various instantaneous gaze-movement angles (the angle between one's gaze and one's direction of linear movement). They are $\mathrm{IM}$, outward deceleration (OD), and outward acceleration (OA). IM is always acceleratory.

Consider a possible sequence of events that might accrue over many seconds for a nonfixated object off one's path. At first, an object may undergo $O A$ as it would in optical flow. Later, because of the increasing gaze-movement angle it may begin a phase of OD, slowing down. As the angle increases further it may start a retrograde motion, $\mathrm{IM}$, and eventually cross through the fovea and undergo $\mathrm{OA}$, this time in the direction opposite from that initially. Now consider the panels in Figure 1 in detail. In each is shown a plan, or bird's eye, view of a quadrant of terrain in front of a moving observer, indicated by a small black square in the lower left corner of the panel. His or her path of movement is linear, and up each left-hand edge. The paths in each panel are the same; the lines of gaze, however, are not. In each, the fixated object is the other small square along the line of gaze.

The upper left panel is a reference situation; it shows projected retinal motions for linear translation with the observer looking in the direction of motion. Thus, by Equation 1, rotational flow is null and retinal flow equals translational (or optical) flow. Notice that all motion to the right of gaze between the direction of movement and $90^{\circ}$ to the side is OA. The same is true for the left forward quadrant, although not shown. Thus, the projected motion of all objects in front of the observer is acceleratory; all that is behind is deceleratory, hence OD. The upper right panel shows motions for an individual looking at an object instantaneously $8^{\circ}$ to the right of the path. Here again, as projected onto the retina, most objects on the terrain undergo $\mathrm{OA}$, but some new regions show other types of motion. In particular, two slivers of space in front of the fixated object show IM and $\mathrm{OD}$; similarly, two larger regions farther away also show them. These areas are enlarged in the lower left panel, showing a gaze-movement angle of $16^{\circ}$; and are larger still in the lower right panel for an angle of $45^{\circ}$.

As one moves linearly through an environment and main- tains fixation on an object off one's path, the gazemovement angle will increase. Thus, for example, on an experimental trial one might start with an angle of $8^{\circ}$ and finish with one of $16^{\circ}$, as in the upper right and lower left panels of Figure 1. During the course of that trial, presenting simulated pursuit fixation in a cluttered environment, different objects off the line of gaze may exhibit IM or OD. These might serve as local information for wayfinding, in addition to DMP. Notice that for DMP to be useful, one need not compute the depth of objects in the field; the fastest moving is likely to be among the nearest. However, for IM and OD to be useful, one must first have a rough depth map of the environment, knowing near from far. Indeed, Vishton and Cutting (1995) suggested that depth is most naturally computed prior to heading, and I will return to this idea later.

\section{Overview}

Cutting et al. (1992) found that DMP, IM, and to some degree OD contributed to wayfinding, and that performance was considerably worse without them. Such results, along with others, form the empirical basis of a theory of wayfinding based on multiple sources of local information in retinal flow, and as such deserve independent replication. Fortunately, the data of Kim et al. (1996) serve this purpose.

\section{More Evidence for the Use of Multiple Sources of Local Information: The Data of Kim et al. (1996)}

Kim et al. (1996) conducted two experiments using stimuli with schematic trees stochastically constrained in an environment. These were patterned after those of Cutting et al. (1992, Experiment 1). A scaled, plan view of the layout is suggested in Figure 2 for a trial with the observer looking right with initial and final gaze-movement angles of $8^{\circ}$ and $16^{\circ}$, respectively. As we did, Kim et al. varied initial gazemovement angles in octave steps between $0.125^{\circ}$ and $8^{\circ}$. Trial sequences simulated the observer's pursuit fixation on a central tree during linear translation. The observer's task was to indicate which side, to the left or right, he or she was headed with respect to his or her gaze. Experiment 1 had two conditions, one in which the stimuli extended the full height of the display, as in Cutting (1986, Experiments 9-11), and the other in which information about relative size and height in the visual field were present as well, as in Cutting et al. I will focus on the latter, which they call their expansion condition. Experiment 2 used stimuli violating the DMP rule of Equation 2. This is an important manipulation, and I will focus on it as well. Before considering the

\footnotetext{
${ }^{4}$ Sometime after Cutting et al. (1992) was published, Charles Chubb helped me determine that aspects of its Figure 13 were incorrect. After reanalyses I then found that the discussion of the analyses in Cutting et al. (Experiments 1 and 2) was nonetheless correct as printed. Because I had already published a correction to Equation 6 (Cutting et al., 1992, p. 129), due to an error introduced by the printer after proof, I was unable to correct the figure. I am pleased to have the opportunity to do so here.
} 


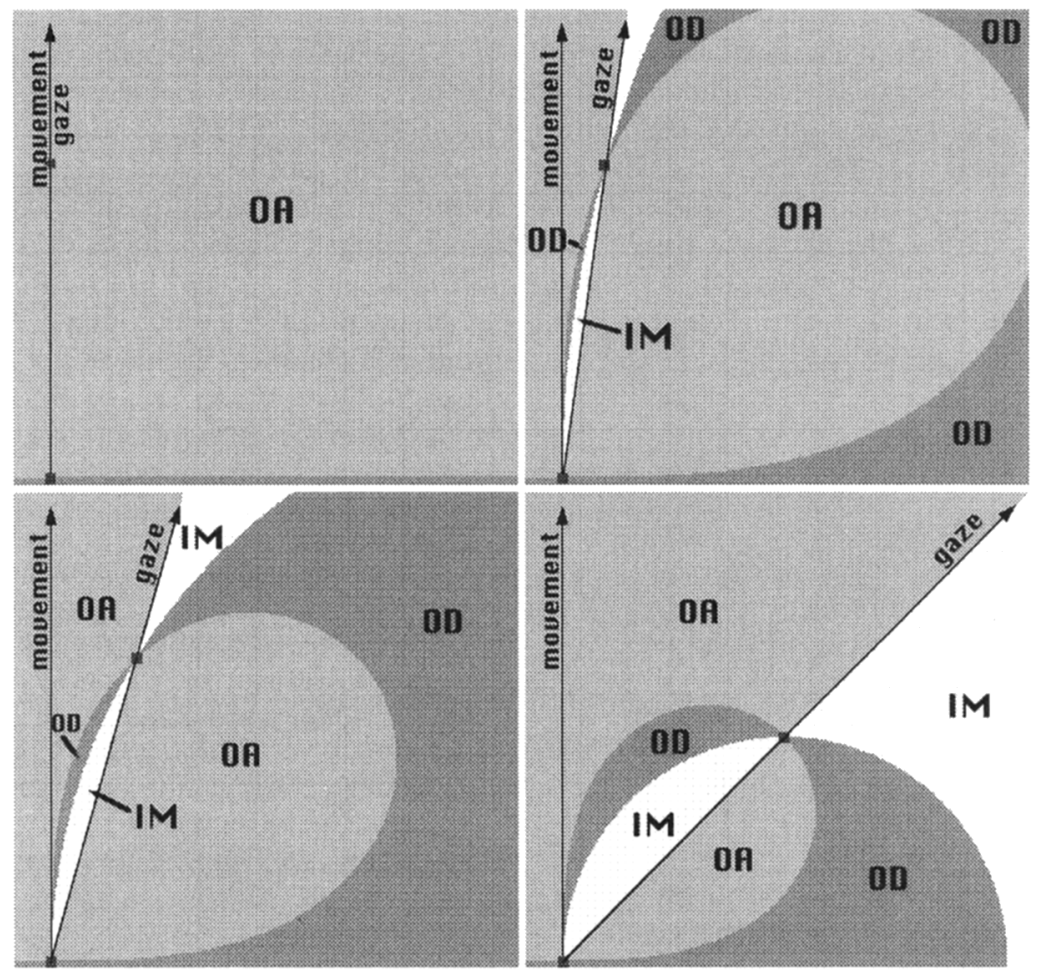

Figure 1. A plan view of the forward-right quadrant of the environment in front of a moving observer. Superimposed on this layout are the patterns of motion for all possible objects as they would be projected in retinal flow when the observer moves forward along a linear path and fixates on a given object in a particular location. Three classes of motion are distinguished-inward displacements (IM), outward acceleration (OA), and outward deceleration (OD). The upper left panel shows fixation along the path of motion, and successive panels show fixations on objects $8^{\circ}$ (upper right panel), $16^{\circ}$ (lower left panel), and $45^{\circ}$ (lower right panel) to the right. This figure supersedes Figure 13 of Cutting et al. (1992). The patterns for differential parallactic displacements are not shown here; instead, see Cutting et al., Figures 3 and 8 ).

data and how they mesh with DMP, IM, and OD, however, let me outline how one calculates these local sources of information.

\section{Calculations}

In general, I have found that the end of these pursuit fixation sequences has more information than any other segment, so in the calculations here the end values are always used; see Cutting et al. (1992, Experiments 1 and 2) and Appendix A for justifications and any difference across a sequence. The calculation procedure follows several steps. First, one must translate and rotate the world coordinates of the trees so that the observer's eye is always at the origin and the heading vector lies along the $z$-axis. Second, because I assume that the eye undergoes pursuit fixation and generating rotation generally in the horizontal plane, the displacements follow the rule

$$
\delta \theta / \delta z=-x /\left(x^{2}+z^{2}\right),
$$

where $\delta \theta / \delta z$ is the lateral angular displacement ( $\theta$ being the angle in the horizontal plane through the eye) of an object due to an incremental step forward along the $z$-axis and, in the $x z$ plane, $x$ is the instantaneous location of the particular object of interest measured laterally and $z$ is its instantaneous location measured in depth. Context and derivation for this equation are given in Cutting et al. (1992, pp. 46 and 129; Cutting, 1986; see also Gordon, 1965). Using it, one can calculate the optical displacements for the seven trees with respect to the heading vector. Third, for each set of calculations, one must subtract the displacement of the foveated tree from those of the other six trees. This operation yields the field of retinal displacements. Fourth, one then determines the displacement with the greatest absolute value among the six nonfoveated trees. If it is against the direction of the heading vector, code the trial as +DMP (1 in a regression); if toward the heading vector, code it as - DMP ( 0 in a regression). Fifth, one must determine if any nonfoveated trees move toward the fovea. In retinal coordinates one or more to the left of the foveated tree must be moving right; one or more to the right must be moving left. If IM is present for any one of the six trees, code the trial as +IM ( 1 in a regression); if absent in all six trees code it $-\mathrm{IM}$ (0 in a regression). Finally, one must recalculate the 


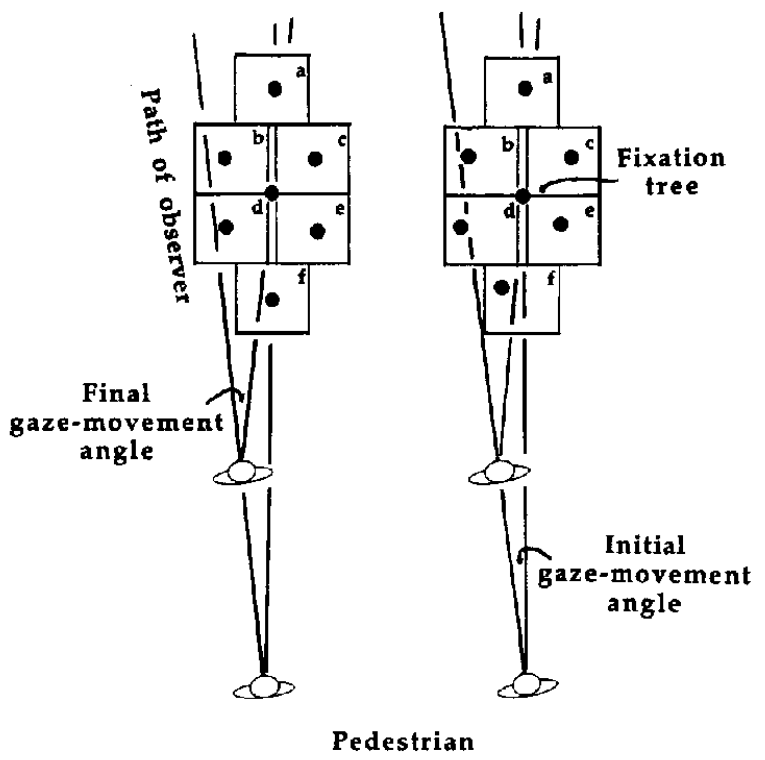

Figure 2. Schematic portrayals of the layout of the seven trees in the two experiments of Kim et al. (1996; Experiment 1 on the left; Experiment 2 on the right) for initial gaze-movement angles of $8^{\circ}$. In both experiments, the central tree was always fixed in position and at the center of the screen throughout the trial. In Experiment 1 the tree positions in the six surrounding areas were stochastically generated within rectangular regions. In the left panel, the median position of these six trees is shown to be the middle of the region in which each was constrained, as one would expect. However, in Experiment 2, in which trials were selected to have misleading differential parallactic displacement (DMP) information, the positions of trees $b, d$, and $f$ are in reliably different locations. Tree $f$ in Experiment 1 would typically generate DMP, but as shown in its position in Experiment 2 would generate inward displacement information.

retinal displacements just before the end of the sequence for all trees. I used the retinal positions $98 \%$ through the sequence, or roughly the values at Frame 382 of Kim et al.'s (1996) 390-frame sequences. If any one of these six absolute displacements are greater than the end-of-sequence values, then one tree is decelerating, and code the trial as $+O D$ ( 1 in a regression). If the displacements of all endof-sequence values are larger, then there is only acceleration, and code the trial as - OD ( 1 in a regression). With these methods in mind, let us consider reanalyses of the Kim et al. data.

\section{Experiment 1}

Overall correct performance in the expansion condition was reasonably good. By the logic and calculations of Cutting et al. (1992; Vishton \& Cutting, 1995), observers moving at $1.85 \mathrm{~m} / \mathrm{s}$ (at an eye height of $1.6 \mathrm{~m}$ ) should attain $95 \%$ performance in the wayfinding task at an initial gazemovement angle of about $4^{\circ}$. When the individual data were fit with logistics functions, 6 of the 8 observers attained this criterion, a result comparable with those of Cutting et al. and Vishton and Cutting. More important in this context, however, are the selected analyses according to the available information sources. The overall method is one of stepwise linear regression, considering first the variance accounted for by DMP, then by IM, and then by OD. Analyses are shown in the upper panels of Figure $3 .^{5}$ Table 1 shows the proportion of trials on which these data are based.

As shown in the upper left panel of Figure 3, performance was reliably better on those trials with DMP than on those without, $F(1,892)=36.08, p<.0001$, as Kim et al. (1996) reported. As in the data of Cutting et al. (1992, Experiments 1 and 2), however, performance also improved on other trials with increasing gaze-movement angles. Kim et al. rightly noted that some other information must account for these results. The upper middle panel of Figure 3 shows the next step. In particular, those trials with predictive DMP, $\mathrm{IM}$, or both are contrasted with those in which neither was present. The pattern of results shown is similar to that of Cutting et al. (Figure 12): Performance on DMP + IM trials was quite good; performance on other trials was much worse. A partial-correlation analysis showed that IM significantly contributed to the results, $F(1,892)=26.97, p<$ .0001 . Moreover, DMP and IM were not highly correlated across the 896 trials in this study $(r=.13)$, just as they were not in those of Cutting et al. (56 trials in Experiment 1 and 3,840 trials in Experiment 2, $r s<.15$ ). The upper right panel of Figure 3 continues the analysis, adding OD to the cluster of information. The perceptual impact of OD was reliable, but less potent than the other two sources of information, $F(1,892)=7.90, p<.005$. Again, OD was not highly correlated with DMP or IM $(r s<.06$ and .16 , respectively). There are some discrepancies between values shown in Figure 3 and Table 1 here, and those of Tables 1 and 2 of Kim et al.; Appendix A addresses these.

Thus, the data of Kim et al. (1996) replicate our previous work. After replication, the next step is logical extension, and their Experiment 2 is a fine example. They argued that if predictive DMP supports wayfinding and if it were not present on all trials, one should be able to select trials with misleading DMP, and performance (should it prove adequate) must then be a function of other sources.

\section{Experiment 2}

The goal of Kim et al. (1996) was to select 112 trials from Experiment 1, 16 at each of the seven gaze-movement

\footnotetext{
${ }^{5}$ The data are plotted as a function of initial gaze-movement angle, rather than final gaze-movement angle (and as in Cutting et al., 1992). The change is due to the logical consideration that Cutting et al. argued: At least $3 \mathrm{~s}$ of stimulation are needed to perform the wayfinding task at reasonable accuracy, and these $3 \mathrm{~s}$ are taken up by the reaction time interval, during which the viewer is looking at the environment. Thus, although less conservative, the initial gaze-movement angle is the more appropriate independent variable. See Vishton and Cutting (1995) for further discussion.
} 


\section{Experiment 1}
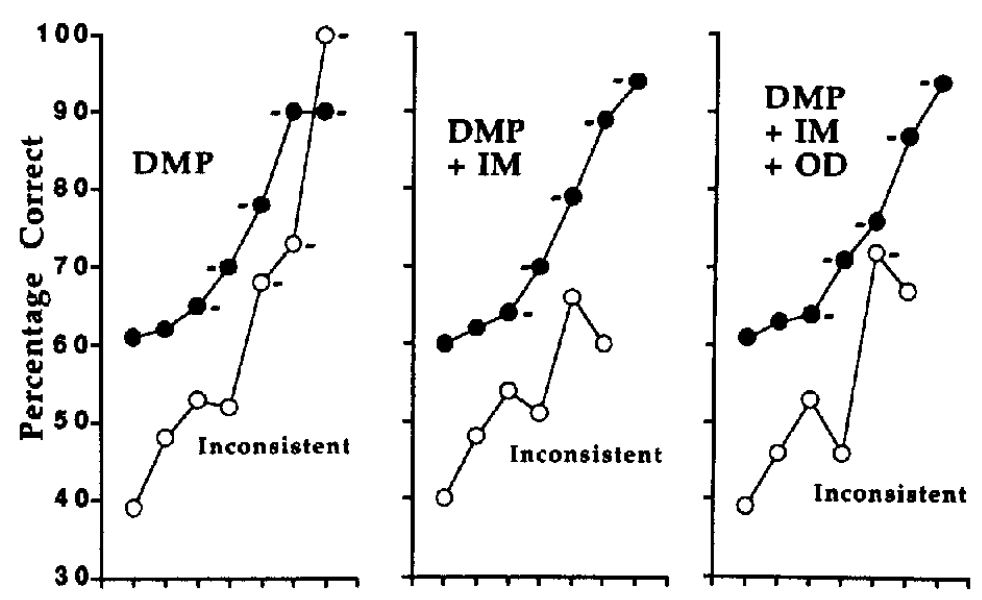

\section{Experiment 2}

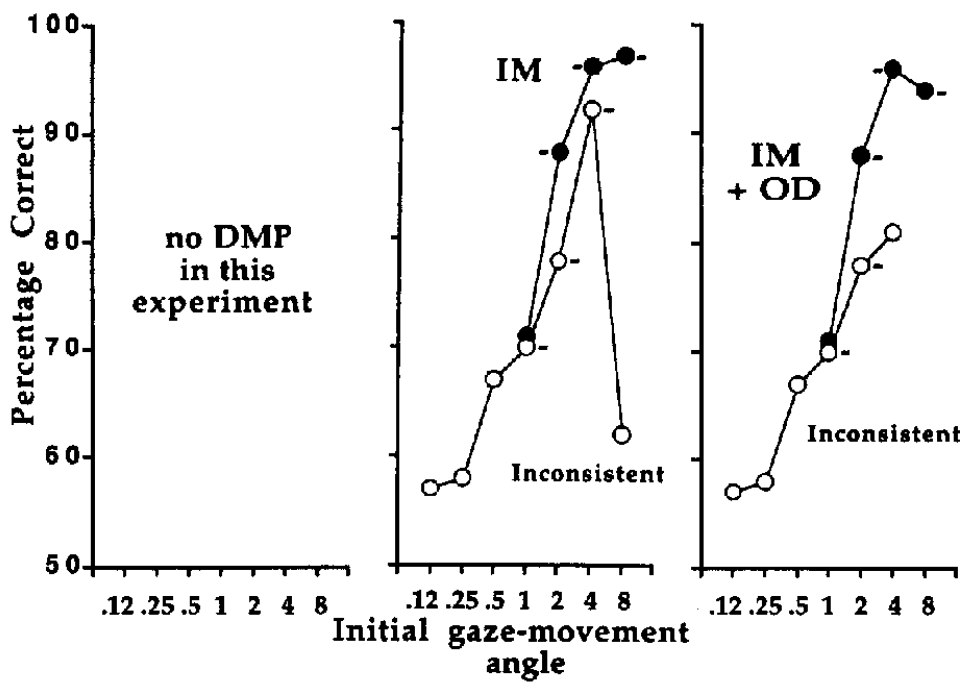

Figure 3. The upper panels show the data from the expansion condition of Experiment 1 of Kim et al. (1996), plotted as a function of initial gaze-movement angle; the lower panels show the data from their Experiment 2. Chance performance is 50\%. Partial correlations show that differential parallactic displacement (DMP), inward displacement (IM), and outward deceleration (OD) each separately contribute to performance in Experiment 1 and that IM and OD both contribute to performance in Experiment 2. Horizontal tick marks indicate data points significantly different from chance $(\alpha<.05)$ when corrected for multiple comparisons. Data points are excluded where they would represent less than $5 \%$ of the data at a particular gaze-movement angle, the same criterion as used by Cutting et al. (1992, Figure 12).

angles, whose tree placements were such that DMP at all points in the sequence would mislead observers. They largely succeeded in their selection: 110 trials had misleading DMP (most rapid motion in the direction of heading, not against it). How such selection is done is suggested in Figure 2. The left panel shows the median positions for the trees in Experiment 1 for the 128 trials with an initial gaze-movement angle of $8^{\circ}$ and the right panel those for the 16 corresponding trials in Experiment 2. ${ }^{6}$ Those for Experiment 1 are nearly in the center of their respective regions, as one would expect. Those for Experiment 2, however, are somewhat different. That is, the trees closest to the observ-

\footnotetext{
${ }^{6}$ The positions of the trees in the stimuli were calculated separately for trials in which the observer's path of movement was to the left and to the right of fixation. Then the positions for the trees during trials looking to the right (as in Figure 2)-ordered $a, b, c$, $d, e$, and $f$-were added to those for trials looking to the leftordered $a, c, b, e, d$, and $f$. After this adjustment, statistical analyses were performed.
} 
Table 1

Proportion of Trials in Each Category of Interest That Are Predictive of Heading Direction at Each Gaze-Movement Angle

\begin{tabular}{|c|c|c|c|c|c|c|c|}
\hline $\begin{array}{l}\text { Initial gaze-movement } \\
\text { angle (degrees) }\end{array}$ & DMP & $\mathbf{I M}$ & $+\mathrm{IM}$ & OD & $+\mathrm{OD}$ & $\begin{array}{l}\text { Other trials } \\
\text { (OA only) }\end{array}$ & DDLO \\
\hline \multicolumn{8}{|c|}{ Experiment $1^{\mathrm{a}}$} \\
\hline 0.125 & .42 & .01 & .01 & .01 & .01 & .56 & .52 \\
\hline 0.25 & .55 & .01 & .00 & .02 & .01 & .44 & .49 \\
\hline 0.5 & .53 & .08 & .03 & .05 & .01 & .43 & .52 \\
\hline 1.0 & .53 & .09 & .02 & .11 & .06 & .38 & .52 \\
\hline 2.0 & .56 & .31 & .14 & .23 & .07 & .23 & .59 \\
\hline 4.0 & .77 & .52 & .12 & .27 & .05 & .07 & .88 \\
\hline 8.0 & .80 & .69 & .17 & .38 & .02 & .01 & .95 \\
\hline \multicolumn{8}{|c|}{ Experiment $2^{b}$} \\
\hline 0.125 & .06 & .00 & .00 & .00 & .00 & .94 & .38 \\
\hline 0.25 & 一 & .00 & .00 & .00 & .00 & 1.00 & .44 \\
\hline 0.5 & - & .00 & .00 & .00 & .00 & 1.00 & 44 \\
\hline 1.0 & .06 & .19 & .19 & .00 & .00 & .75 & .56 \\
\hline 2.0 & - & .37 & .37 & .00 & .00 & .63 & .50 \\
\hline 4.0 & - & .63 & .63 & 31 & .25 & .12 & .50 \\
\hline 8.0 & - & .94 & .94 & .39 & .06 & .00 & .75 \\
\hline
\end{tabular}

Note. DMP $=$ differential parallactic displacements; $\mathrm{IM}=$ inward displacements; $+\mathrm{IM}=$ the number of trials in which IM was present and DMP was not; OD = outward deceleration; $+\mathrm{OD}=$ the number of trials in which OD was present and both IM and DMP were not; OA = outward acceleration. Thus, the columns DMP, +IM, +OD, and OA-only add to 1.0. See Figure 1 for examples of locations of non-DMP sources of information in an environment as it would project onto a retinal flow field; see Cutting et al. (1992, Figures 3 and 8 ) for discussions of DMP. DDLO $=$ displacement direction of the largest object in the visual field. Dashes indicate no data.

${ }^{\mathrm{a}} n=128 .{ }^{\mathrm{b}} n=16$.

er's path- $-b, d$, and $f$-were in statistically different locales in either or both of their $x z$ coordinates, $t \mathrm{~s}(142)>1.98$, $p s<.05$. In particular, Tree $f$, which might normally generate DMP because it would be the fastest moving in the visual field, would now generate IM. Note also from the patterns in Table 1 that the general result of removing predictive DMP was to increase the proportion of trials with IM, but not that for OD.

Overall, observers again performed reasonably well. Individual performance functions were fit to logistics curves and again 6 out of 8 observers met the $95 \%$ performance criterion at an initial gaze-movement angle of $4^{\circ}$. The stimuli and results were next parsed as before. As shown in the lower middle panel of Figure 3, performance was better on those trials with IM than on those without, $F(1,108)=$ $31.6, p<.0001$. Moreover, when IM + OD were considered in consort in the lower right panel, this difference increased somewhat, demonstrating again that $O D$ aided performance, $F(1,108)=5.3, p<.03$. Again, as in Experiment 1, IM and OD were not correlated across the 112 trials $(r=.13)$.

\section{Overview}

The data of Experiment 1 are consistent with the idea that three sources of local motion information-DMP, IM, and OD-are used for wayfinding by observers; the data of Experiment 2 suggest that when predictive DMP is removed, IM (but not OD) information increases and is used. Thus, despite the conclusions of Kim et al. (1996), both my theory of wayfinding as given in Cutting et al. (1992) and the metatheoretical position of directed perception are corroborated. Appendix $B$ addresses their conclusions.

Beyond corroboration, however, scrutiny of the results in the right panels of Figure 3 suggests there may be some residual information, not yet isolated, at initial gazemovement angles of $1^{\circ}$ and $2^{\circ}$ that may yet serve wayfinding. Performance on these OA-only trials was above chance, although reliably poorer than on DMP + IM + OD trials. Directed perception dictates that researchers must not satisfice (Cutting, 1986, 1991b), but must continue to look for perceptual information that may serve a task. The final section addresses some of the possibilities and will show that another source of local information must replace DMP in the consideration of wayfinding.

\section{Other Parallaxlike Information in Retinal Flow: Global and Local Analyses}

The idea that at least three sources of local informationDMP, IM, and OD-may be used for wayfinding is not satisfying to all researchers. As a theory of wayfinding, such a list of available and used information may seem inelegant at best and ad hoc at worst. Metatheoretical predilections aside, one might ask, Could it not be that there is a single source of information that captures all the important variance in the data? Here I will consider four other candidates; Table 2 summarizes their attributes in comparison with DMP. First consider three global sources of information. 
Table 2

Five Related Sources of Parallactic Information and How They Differ

\begin{tabular}{|c|c|c|c|}
\hline $\begin{array}{l}\text { Characterization of parallax } \\
\text { information }\end{array}$ & $\begin{array}{l}\text { Formalism in } \\
\text { this context }\end{array}$ & Description & $\begin{array}{c}\text { Information } \\
\text { type }\end{array}$ \\
\hline $\begin{array}{l}\text { Differential parallactic displacements } \\
\text { (DMP; Cutting, 1986; Cutting et } \\
\text { al., 1992) }\end{array}$ & $-\max \left(\mathbf{v}_{i}\right)$ & $\begin{array}{l}\text { Heading direction is opposite to the most rapidly } \\
\text { moving object in the retinal field. }\end{array}$ & Local \\
\hline $\begin{array}{l}\text { Differential motion (Rieger \& } \\
\text { Lawton, 1985) }\end{array}$ & $-\Sigma\left(v_{1}^{2}\right)$ & $\begin{array}{l}\text { Heading direction is opposite to the sum of the } \\
\text { squared vectors in a region of the retinal field. }\end{array}$ & Global \\
\hline $\begin{array}{l}\text { Spatial pooling (see Warren \& } \\
\text { Saunders, 1995) }\end{array}$ & $-\Sigma\left(\mathbf{v}_{i}\right)$ & $\begin{array}{l}\text { Heading direction is opposite to the sum of the } \\
\text { vectors. }\end{array}$ & Global \\
\hline Size-weighted spatial pooling & $-\Sigma\left(\omega_{i} \mathbf{v}_{i}\right)$ & $\begin{array}{l}\text { Heading direction is opposite to the weighted } \\
\text { sum of the vectors in a region, where the } \\
\text { weights are determined by the relative retinal } \\
\text { size of each moving object. }\end{array}$ & Global \\
\hline $\begin{array}{l}\text { Displacement direction of the largest } \\
\text { object (DDLO) }\end{array}$ & $-\mathbf{v}\left(O_{\max }\right)$ & $\begin{array}{l}\text { Heading direction is opposite to motion of the } \\
\text { largest object in the field. }\end{array}$ & Local \\
\hline
\end{tabular}

\section{Global Source 1: Differential Motion}

As suggested earlier there have been many characterizations and models of the motion generated by the translation and rotation of an observer's eye. One was suggested by Rieger and Lawton (1985; Rieger \& Toet, 1985). These authors showed that, in our context and within a suitably small region of retinal space, the difference vector of the sum of all the squared vectors would point in the direction of heading. Sampling several such regions should yield a good estimate of the aimpoint. Warren et al. (1988; Warren, 1995) called this differential motion, Hildreth (1992) adapted this procedure, and Heeger and Jepson $(1990,1992)$ used it as a benchmark to test their own theory. ${ }^{7}$ Thus, it has received widespread recognition. Rieger and Lawton chose a squared-vectors approach so that "as desired, the larger difference vectors ... within a neighborhood will essentially determine" (p. 355) the direction of heading. Effectively, differential motion is a nonlinear pooling of motions within a region of retinal space. In our context, with the direction of heading either to the left or right of fixation, a reduced version of the formalism that captures differential motion is given in Table 2.

To use this algorithm, I assumed that the fixation tree was the center of the retinal field of interest and calculated the six difference vectors and the sum of their squares at the end of the trial sequences of Kim et al. (1996). I then coded each trial $(1$ or 0$)$ with respect to whether the summed difference vector was predictive of the heading, and performed the multiple regression as before. As shown in the upper left panel of Figure 4, differential motion accounted for substantial variance in Experiment $1, F(1,892)=74.5, p<$ .0001 , but even on trials in which differential motion did not predict correct performance, it rose substantially above chance at initial gaze-movement angles of $4^{\circ}$ and $8^{\circ}$. As before, further regression analyses showed that this performance was largely accounted for by $\operatorname{IM}, F(1,892)=29.0$, $p<.0001$, and perhaps somewhat by $\mathrm{OD}, F(1,892)=2.48$, $p<.01$. Indeed, differential motion acted much like DMP, and because in Experiment 1 it was highly correlated with DMP $(r=.71)$ and not with IM and OD $(r s<.15)$, this is not a surprise; moreover, across the various gaze-movement angles differential motion accounted for no better performance than did DMP, $t(5)<1.5$. In Experiment 2, in which DMP was removed, predictive differential motion was largely removed as well. When it existed it occurred only at initial gaze-movement angles of $1^{\circ}$ or less, and in no case did it reliably account for any data, as shown in the lower left panel of Figure 4. Thus, consideration of this global source, differential motion, has no advantage over the local source, DMP. Moreover, multiple sources of information appear to be used in either case.

\section{Global Source 2: Spatial Pooling}

A second procedure was then investigated, modeled on the simpler idea that all the vectors within the relevant region might be pooled (Perrone \& Stone, 1994; Warren \& Saunders, 1995). In our context the direction of heading from the fixation tree is given by the difference vector of the simple sum of the vectors, as suggested in Table 2. I computed these difference vectors, coded them as to whether they predicted the heading direction, and reran the regression. As shown in the second upper panel of Figure 4, the difference vector from spatial pooling was a good predictor of performance in Experiment $1, F(1,892)=52.9$, $p<.0001$, but it too left a residue of trials in which performance was above chance, and this residue was largely accounted for by $\mathrm{IM}, F(1,892)=32.5, p<.0001$. OD was not a reliable predictor in this context. Again, spatial pooling was correlated with DMP $(r=.45)$ and not with IM; spatial pooling accounted for little variance in the results of

\footnotetext{
${ }^{7}$ Heeger and Jepson (1990, 1992) devised a model in which depth, rotation, and translation are computed and minimized simultaneously. This is a brute force method, and interestingly it performs no better than that of Rieger and Lawton (1985) within the range of gaze-movement angles considered here.
} 


\section{Experiment 1}

\section{Differential} motion
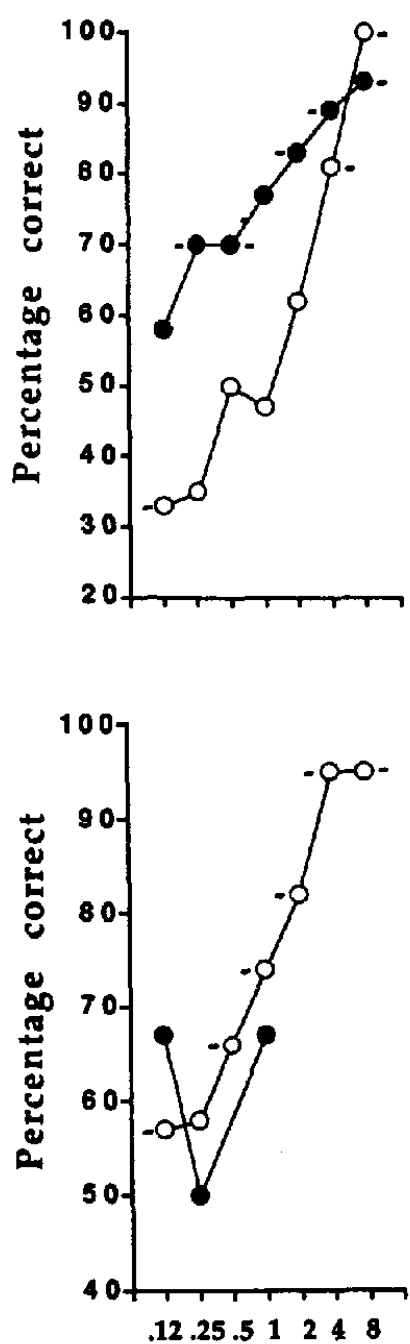

\section{Spatial pooling}

Displacement direction of the largest object

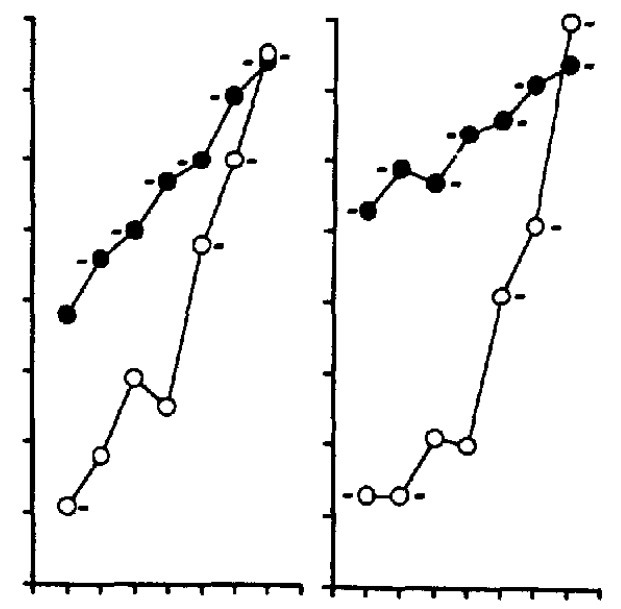

Experiment 2

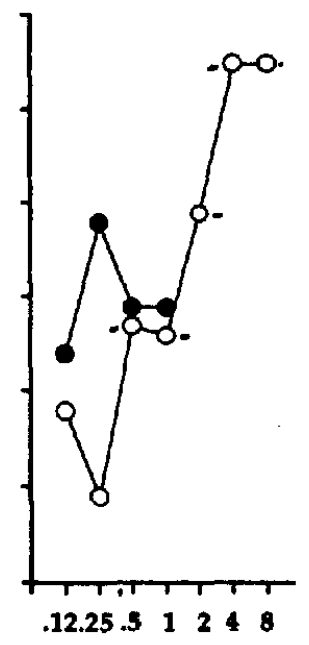

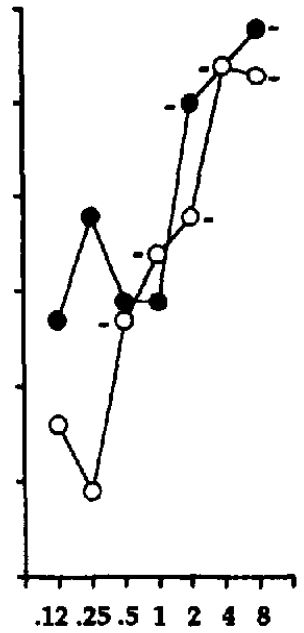
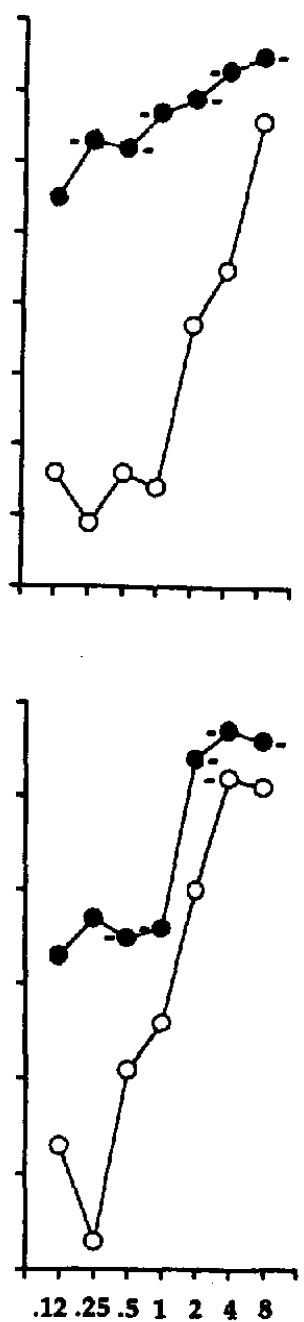

\section{Initial gaze-movement angle}

Figure 4. The results of applying four other parallax computations to the results of the two experiments of Kim et al. (1996). Functions with filled circles represent the data consistent with each hypothesis in question; those with unfilled circles represent data inconsistent with each hypothesis. In all cases in both experiments residual information in the stimuli at large gaze-movement angles suggests that a single source of information is inadequate to account for the data. Again, horizontal tick marks indicate data points significantly different from chance. Data points are excluded where they would represent less than $10 \%$ of the data at a particular gaze-movement angle.

Experiment 2, as shown in the second lower panel of Figure 4 , and its performance at the various gaze-movement angles was not reliably different than those of DMP, $t(5)<1.6$. Again, consideration of a global source, here spatial pooling, provided no advance over DMP, and again multiple sources of information would appear to be used in either case.

\section{Global Source 3: Size-Weighted Spatial Pooling}

Next, I considered a third procedure. Differential motion and spatial pooling models are typically applied to points within a flow field, and because most stimuli used in this research domain are dot fields, this analysis seems pertinent. However, the stimuli of Kim et al. (1996)-modeling my 


\section{Experiment 1}
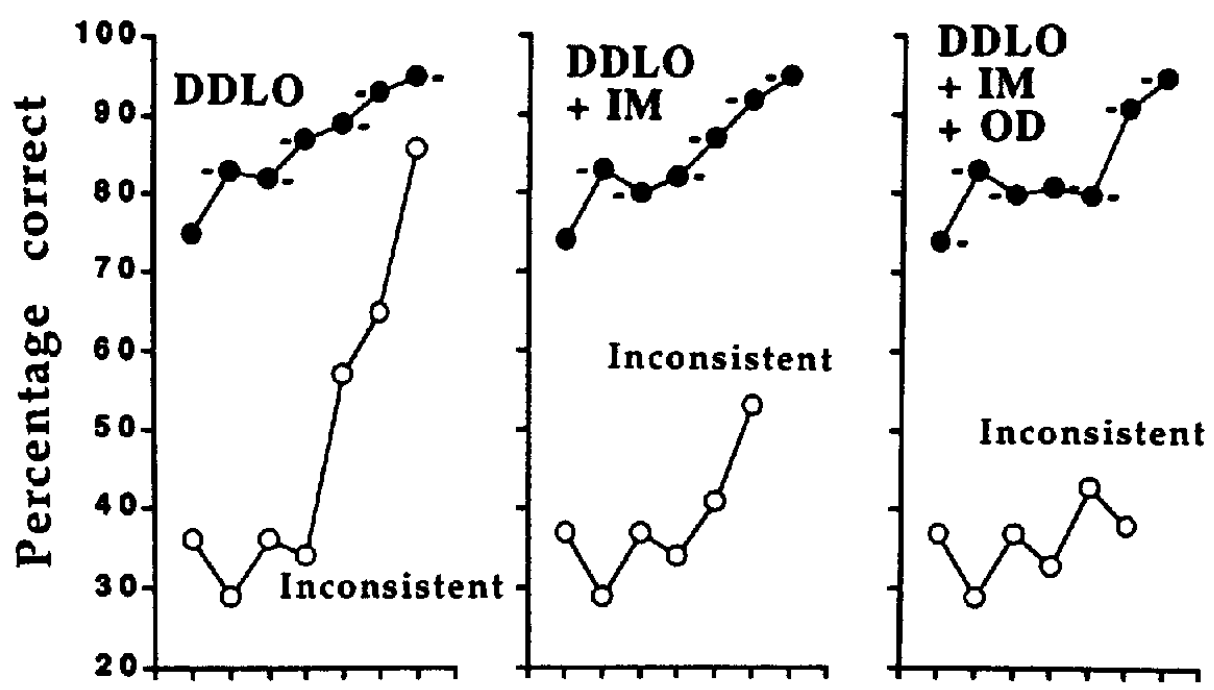

\section{Experiment 2}
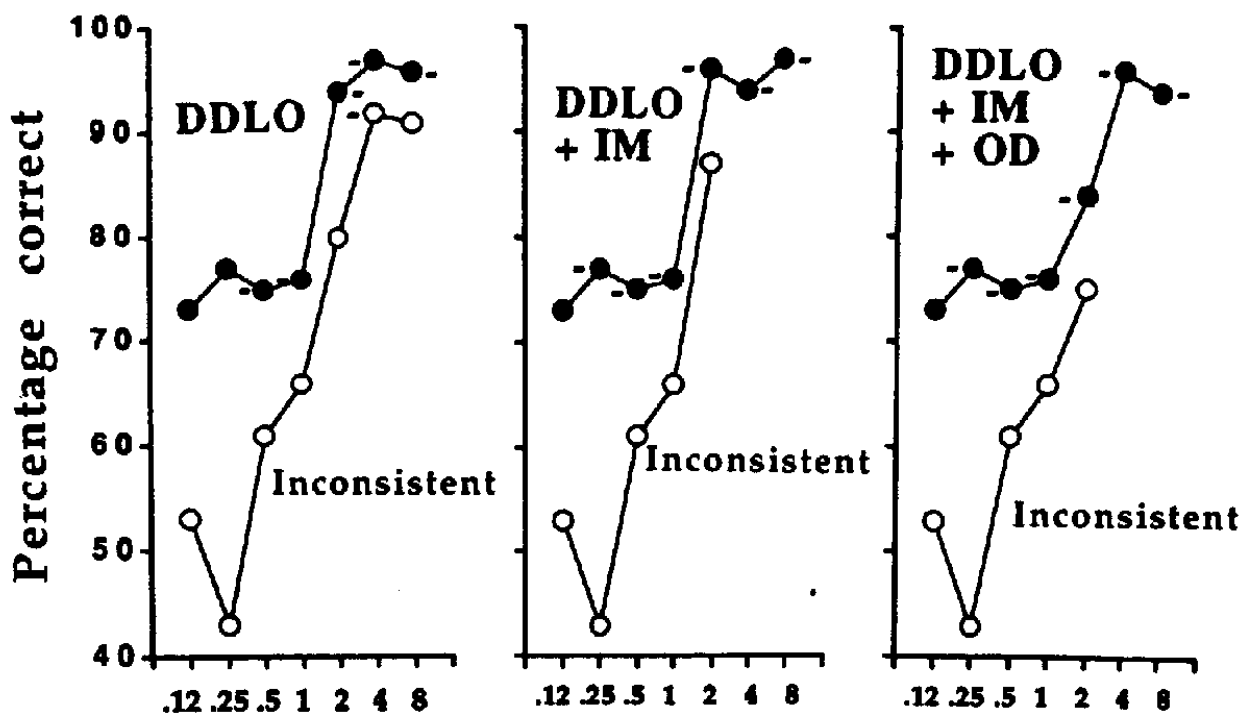

\section{Initial gaze-movement angle}

Figure 5. The application of the displacement direction of the largest object (DDLO) in the visual field as a predictor of the data in Experiments 1 and 2 of Kim et al. (1996). The panels of this figure parallel those of Figure 3, and show that three sources of local motion information-DDLO, inward displacement (IM), and outward deceleration (OD) - are needed to account for the data. Again, horizontal tick marks indicate data points significantly different from chance. Data points are excluded where they would represent less than $5 \%$ of the data at a particular gaze-movement angle.

own (Cutting, 1986; Cutting et al., 1992)—were extended objects. Because most of Kim et al.'s stimuli were lines that extended in their length as they approached the observer, I decided to weight each vector in the spatial pooling model by the size of the object to which it was attached, and again I reran the regression. This weighting procedure increases the significance of those objects that are close to the observer.

As shown in the third upper panel of Figure 4, for Experiment 1 size-weighted spatial pooling accounted for substantial variance, $F(1,892)=150.5, p<.0001$, and provided a significantly improved set of predictions over DMP, 
differential motion, and simple spatial pooling, $t \mathrm{~s}(5)>2.85$, $p s<.03$. Moreover, it was a reliable predictor of performance in Experiment 2 as well, $F(1,108)=2.62, p<.01$, as shown in the third lower panel. Nonetheless, and as before, in both experiments there was residual information best accounted for by IM, $F(1,892)=23.2, p<.0001 ; F(1$, $108)=23.7, p<.0001$, and $\mathrm{OD}, F(1,892)=7.9, p<.007$; $F(1,108)=1.85, p<.07$, in Experiments 1 and 2, respectively. Thus, here we have a global information source that is superior to DMP, but that still must be coupled with one or two local sources to account for the performance data.

\section{Local Source 2: The Displacement Direction of the Largest Object in the Field}

Given the success of the third global measure, which considered the size of each object in the retinal field, I decided to investigate further the effects of size. The simplest case is to consider the displacement direction of the largest (or nearest) object (DDLO) in the stimulus array. Thus, whereas DMP was the first local parallax source considered, DDLO is the second. The prediction is that heading should be opposite DDLO. All trials in Experiments 1 and 2 were reanalyzed for the retinal motions of Tree $f$ in Figure 2, which would always be the largest object in the stimulus (coded appropriately), and the regression was performed for a last time. As shown in the upper right panel of Figure 3, DDLO was an excellent predictor of performance in Experiment $1, F(1,892)=277.0, p<$ .0001 , considerably superior even to the size-weighted spatial pooling measure, $t(5)=5.3, p<.001$. Again, there appeared to be residual information used at larger gazemovement angles, and this seems to be accounted for by IM and OD, $F(1,892)=4.2, p<.04$, and $F(1,892)=9.6$, $p<.002$, respectively. The power of DDLO was also shown in Experiment 2. Again, it was a strong predictor of the data, $F(1,108)=27.7, p<.0001$, aided by $\mathrm{IM}, F(1$, $108)=22.4, p<.0001$, but not OD. Figure 5 shows the titration of effects for DDLO, IM, and OD, mirroring those in Figure 3. Notice that, unlike Figure 3, performance is nowhere above chance when some aspect of this complex of information is not present. Table 1 also gives the proportion of trials in which DDLO is predictive of heading direction.

Thus, DDLO, a local source of motion information, is superior to DMP and all three global sources. Moreover, DDLO appears to be combined with IM and perhaps OD to aid in wayfinding performance. Consider two sidelights. First, one might wonder why Cutting et al. (1992) did not detect the usefulness of DDLO. One reason is that in their Experiment 2, DDLO and DMP were perfectly correlated because of the nature of the configuration of nonfixation trees; in addition, in their Experiment 1 there were simply too few trials to distinguish the two. Only with the large number of trials investigated by Kim et al. (1996) could the two be separated. Thus, I applaud Kim et al. for forcing this important revision of my theory. Second, DDLO remains, as DMP did before it, a source of information for wayfinding that does not necessitate the computation of depth for it to be used; for IM and OD depth determination remains a necessity. However, DDLO is a local source of information that relies on naturally occurring size-distance relations. Thus, the relationship of these three local sources is tighter than in previous theory (Cutting et al., 1992).

\section{Overview}

Considering five parallaxlike motion sources of information revealed a family of closely related measures: DMP, differential motion, spatial pooling, size-weighted spatial pooling, and DDLO. DMP and DDLO are local measures in the retinal flow field; that is, they rely on the motion of a single element among many. In contrast, the other three are global measures, relying on combinations and joint comparisons of motions within a retinal region. The median intercorrelation for these measures in the data of Kim et al. (1996, Experiment 1) was high $(r=.63)$. Of these, however, the new local source of information-DDLO-was consistently better than the others, but with the others it could not account for accurate performance on all trials. IM, and to a lesser extent $\mathrm{OD}$, continued to prove useful. It remains possible that there is a single source of information that can account for these data, but in consideration of those sources investigated here, that possibility now seems less likely.

\section{Summary}

Reanalyses of the data of Kim et al. (1996) showed that they replicated previous results of Cutting et al. (1992; Cutting, 1986). In particular, three local sources of retinal information appeared to be used by observers to determine the direction of their heading. In pursuing the best manner in which to account for these data, I compared these previous three measures-DMP, IM, and OD-against four others, two of which were taken from the wayfinding literature. Results suggest that three local measures-now DDLO, IM, and $\mathrm{OD}$-are still necessary to account for the data, and that the global measures do not do as well. Thus, given this analysis, it still appears that multiple sources of local information in retinal flow are used by observers to determine the direction of their heading.

\section{References}

Berthoz, A., Israël, I., Georges-François, P., Grasso, R., \& Tsuzuku, T. (1995, July 7). Spatial memory of body linear displacement: What is being stored? Science, 269, 95-98.

Burton, G., \& Turvey, M. T. (1990). Perceiving lengths of rods that are held but not wielded. Ecological Psychology, 2, 295324.

Crowell, J. A., \& Banks, M. S. (1993). Perceiving heading with different retinal regions of optic flow. Perception \& Psychophysics, 53, 325-337.

Cutting, J. E. (1986). Perception with an eye for motion. Cambridge, MA: MIT Press.

Cutting, J. E. (1991a). Four ways to reject directed perception. Ecological Psychology, 3, 25-34. 
Cutting, J. E. (1991b). Why our stimuli look as they do. In G. Lockhead \& J. R. Pomerantz (Eds.), Perception of structure: Essays in honor of Wendell R. Garner (pp. 41-52). Washington, DC: American Psychological Association.

Cutting, J. E., Springer, K., Braren, P. A., \& Johnson, S. H. (1992). Wayfinding on foot from information in retinal, not optical, flow. Journal of Experimental Psychology: General, 102, 41$72,129$.

Cutting, J. E., \& Vishton, P. M. (1995). Perceiving layout and knowing distances: The integration, relative potency, and contextual use of different information about depth. In W. Epstein \& S. Rogers (Eds.), Handbook of perception and cognition: Vol. 5. Perception of space and motion (pp. 69-117). San Diego, CA: Academic Press.

Cutting, J. E., Vishton, P. M., \& Braren, P. A. (1995). How we avoid collisions with stationary and moving obstacles. Psychological Review, 102, 627-651.

Cutting, J. E., Vishton, P. M., Flückiger, M., Baumberger, B., \& Gerndt, J. (in press). Heading and path information from retinal flow in naturalistic environments. Perception \& Psychophysics.

Gibson, J. J. (1966). The senses considered as perceptual systems. Boston: Houghton Mifflin.

Gibson, J. J. (1979). The ecological approach to visual perception. Boston: Houghton Mifflin.

Gibson, J. J., Olum, P., \& Rosenblatt, F. (1955). Parallax and perspective during aircraft landings. American Journal of Psychology, 68, 373-385.

Gilden, D. L., \& Proffitt, D. R. (1989). Understanding collision dynamics. Journal of Experimental Psychology: Human Perception and Performance, 15, 372-383.

Gordon, D. A. (1965). Static and dynamic visual fields in human space perception. Journal of the Optical Society of America, 55, 1296-1303.

Heeger, D. J., \& Jepson, A. D. (1990). Visual perception of threedimensional motion. Neural Computation, 2, 129-139.

Heeger, D. J., \& Jepson, A. D. (1992). Subspace methods for recovering rigid motion I: Algorithm and implementation. International Journal of Computer Vision, 7, 95-117.

Hildreth, E. (1992). Recovering heading for visually-guided navigation. Vision Research, 32, 1177-1192.

Kim, N.-G., Growney, R., \& Turvey, M. T. (1996). Optical flow not retinal flow is the basis of wayfinding by foot. Journal of Experimental Psychology: Human Perception and Performance, $22,1279-1288$.

Maloney, L. T., \& Landy, M. S. (1989). A statistical framework for robust fusion of depth information. Visual Communication \& Image Processing, IV: Proceedings of the SPIE, 1199, 11541163.
Massaro, D. W. (1987). Speech perception by ear and by eye. Hillsdale, NJ: Erlbaum.

Perrone, J. A., \& Stone, L. S. (1994). A model of self-motion estimation within primate extrastriate visual cortex. Vision Research, 34, 2917-2938.

Regan, D. M., \& Beverley, K. I. (1982, January 8). How do we avoid confounding the direction we are looking with the direction we are going? Science, 215, 194-196.

Rieger, J. H., \& Lawton, D. T. (1985). Processing differential image motion. Journal of the Optical Society A, 2, 354-360.

Rieger, J. E., \& Toet, L. (1985). Human visual navigation in the presence of 3D rotations. Biological Cybernetics, 52, 377-381.

Royden, C. S., Banks, M. S., \& Crowell, J. A. (1992). The perception of heading during eye movements. Nature, 360, 583-585.

Van den Berg, A. V. (1992). Robustness of perception of heading from optic flow. Vision Research, 32, 1285-1296.

Van den Berg, A. V., \& Brenner, E. (1994a). Humans combine the optic flow with static depth cues for robust perception of heading. Vision Research, 34, 2153-2167.

Van den Berg, A. V., \& Brenner, E. (1994b). Why two eyes are better than one for judgments of heading. Nature, 371, 700702 .

Vishton, P. M., \& Cutting, J. E. (1995). Wayfinding, displacements, and mental maps: Velocity fields are not typically used to determine one's heading. Journal of Experimental Psychology: Human Perception and Performance, 21, 978-995.

Vishton, P. M., Nijhawan, R., \& Cutting, J. E. (1994). Moving observers utilize static depth cues in determining their direction of motion. Investigative Ophthalmology \& Visual Science, 35 , 2000.

Warren, W. H. (1995). Self motion: Visual perception and visual control. In W. Epstein \& S. Rogers (Eds.), Handbook of perception and cognition: Vol. 5. Perception of space and motion (pp. 263-325). San Diego, CA: Academic Press.

Warren, W. H., \& Hannon, D. J. (1990). Eye movements and optical flow. Journal of the Optical Society of America A, 7, $160-169$.

Warren, W. H., \& Kurtz, K. J. (1992). The role of central and peripheral vision in perceiving direction of self-motion. Perception \& Psychophysics, 51, 443-545.

Warren, W. H., Morris, M. W., \& Kalish, M. (1988). Perception of translational heading from optical flow. Journal of Experimental Psychology: Human Perception and Performance, 14, 644660.

Warren, W. H., \& Saunders, J. A. (1995). Perceiving heading in the presence of moving objects. Perception, 24, 315-331. 


\section{Appendix A}

Expectations for the Frequencies of IM, OD, and OA

How should one assess the values for the occurrence of IM, OD, and OA-only in Table 1 ? One approach is a formal analysis, computing the a priori expectations for each of these variables. Compare Figures 1 and 2 . Figure 1 shows the entire forward left quadrant of the terrain. The superimposition of the grid in Figure 2 on these regions is the essence of the calculations to follow. Given a reasonably large number of trials (satisfied by Experiment 1 of Kim et al., 1996, $n=896$ ), the probability of any given tree generating IM, OD, or OA on a given trial is equal to the proportion of the area within the constrained region that would yield IM, $\mathrm{OD}$, or $\mathrm{OA}$ were a tree placed there. This area can be assessed computationally, based on Equation 3 (also Equation 7 of Cutting et al., 1992) and on the procedure outlined in the body of this Observation. Table A1 shows these proportions for each area in the six tree regions, $a$ through $f$ (as shown in Figure 2), at the beginning and at the end of each gaze-movement angle condition.

Notice first that there is little difference between the beginning and the end value of each type of trial. Information tends to be preserved, and when it is not it follows the order $O A \rightarrow O D \rightarrow I M$ and eventually $\rightarrow \mathrm{OA}$. In particular, when the observer moves forward fixating an object off his path, his or her translation and eye rotation typically starts with some, and creates more, information in IM and OD categories. In all cases this information either preexists at the beginning of the sequence or it is created out of the OA category. Analyses in the body of the Observation indicate that $O A$ is not information.

\section{IM Trials}

Consider first the occurrences of $\mathrm{IM}$ at the end of the sequences across all gaze-movement angles. Using the means at the bottom of Table A1, the a priori probability of an IM trial in the stimulus set would be 1.0 minus the product of all $(1-\mathrm{IM})$ probabilities for each of the six trees, $a$ through $f: p(\mathrm{IM})=1-(.85 \cdot 1.0 \cdot$ $.98 \cdot 1.0 \cdot 1.0 \cdot .94)=1-.78=.22$.

Across all gaze-movement angles the observed probability of an IM trial can be found as the mean of column 3, Table 1 (Experiment 1): This value is .24 , a reasonable fit with expectations. Consider next Table 2 of $\mathrm{Kim}$ et al. (1996). They listed five columns of data, and there are seven possible combinations of $\mathrm{IM}$, $\mathrm{OA}$, and $\mathrm{OD}$ (each singly, three pairs, and all three). I assume from their presentation that there were no $I M+O D$ or IM $+O A$ trials by their calculations. Thus, their idea of the occurrence of IM would be the sum of the means of columns 2 and 6 . Assuming eight trials per participant at each gaze-movement angle, this sum should be divided by 8 . This yields an observed probability of .26, also a reasonable fit.

\section{OD Trials}

Again from Table A1, and mirroring the analysis for IM, the a priori probability of an OD trial would be 1.0 minus the product of

Table A1

A Priori Probabilities $(\times 100)$ of $I M, O D$, and $O A$ at the Beginnings and Ends of Trial Sequences for Each of the Six Nonfoveal Trees at Each of the Seven Initial Gaze-Movement Angles (GMA), Neglecting Consideration of Differential Parallactic Displacement

\begin{tabular}{|c|c|c|c|c|c|c|c|c|c|c|c|c|c|c|c|c|c|c|}
\hline \multirow{2}{*}{$\begin{array}{c}\text { GMA } \\
\text { (degrees) }\end{array}$} & \multicolumn{3}{|c|}{ Farthest tree (a) } & \multicolumn{3}{|c|}{$\begin{array}{l}\text { Far tree on the } \\
\text { heading vector } \\
\text { side }(b)\end{array}$} & \multicolumn{3}{|c|}{$\begin{array}{c}\text { Far tree away } \\
\text { from the heading } \\
\text { vector }(c)\end{array}$} & \multicolumn{3}{|c|}{$\begin{array}{l}\text { Near tree on the } \\
\text { heading vector } \\
\text { side }(d)\end{array}$} & \multicolumn{3}{|c|}{$\begin{array}{l}\text { Near tree away } \\
\text { from heading } \\
\text { vector }(e)\end{array}$} & \multicolumn{3}{|c|}{ Nearest tree $(f)$} \\
\hline & IM & OD & $\mathrm{OA}$ & $\mathbf{M M}$ & OD & $\mathrm{OA}$ & IM & OD & $\mathrm{OA}$ & IM & OD & $\mathrm{OA}$ & IM & OD & OA & IM & OD & $\mathrm{OA}$ \\
\hline \multicolumn{19}{|c|}{ Beginning of trials } \\
\hline 0.125 & 0 & 0 & 100 & 0 & 0 & 100 & 0 & 0 & 100 & 0 & 0 & 100 & 0 & 0 & 100 & 0 & 0 & 100 \\
\hline 0.25 & 0 & 0 & 100 & 0 & 0 & 100 & 0 & 0 & 100 & 0 & 0 & 100 & 0 & 0 & 100 & 0 & 0 & 100 \\
\hline 0.5 & 0 & 2 & 98 & 0 & 0 & 100 & 0 & 0 & 100 & 0 & 0 & 100 & 0 & 0 & 100 & 1 & 2 & 97 \\
\hline 4.0 & 13 & 17 & 70 & 0 & 0 & 100 & 0 & 3 & 97 & 0 & 0 & 100 & 0 & 0 & 100 & 8 & 6 & 86 \\
\hline 8.0 & 27 & 16 & 57 & 0 & 0 & 100 & 2 & 9 & 88 & 0 & 2 & 98 & 0 & 0 & 100 & 17 & 13 & 70 \\
\hline $\mathrm{M}$ & 7 & 7 & 86 & 0 & 0 & 100 & 0 & 2 & 98 & 0 & 0 & 100 & 0 & 0 & 100 & 4 & 4 & 92 \\
\hline \multicolumn{19}{|c|}{ End of trials } \\
\hline 0.125 & 1 & 2 & 97 & 0 & 0 & 100 & 0 & 0 & 100 & 0 & 0 & 100 & 0 & 0 & 100 & 0 & 0 & 100 \\
\hline 0.25 & 1 & 3 & 96 & 0 & 0 & 100 & 0 & 0 & 100 & 0 & 0 & 100 & 0 & 0 & 100 & 0 & 0 & 100 \\
\hline 8.0 & 43 & 0 & 57 & 0 & 0 & 100 & 14 & 48 & 58 & 2 & 10 & 88 & 0 & 0 & 100 & 24 & 12 & 64 \\
\hline $\mathbf{M}$ & 15 & 7 & 79 & 0 & 0 & 100 & 2 & 9 & 89 & 0 & 1 & 99 & 0 & 0 & 100 & 6 & 3 & 91 \\
\hline
\end{tabular}

Note. $\quad \mathrm{IM}=$ inward displacements; $\mathrm{OD}=$ outward deceleration; $\mathrm{OA}=$ outward acceleration. 
all six $(1-$ OD) probabilities: $p(\mathrm{OD})=1-(.93 \cdot 1.0 \cdot .91 \cdot$ $.99 \cdot 1.0 \cdot .97)=1-.81=.19$. Across all gaze-movement angles in Table 1 the observed probability of an OD trial (mean of column 5) is .16; again, a reasonable fit. From Table 2 of Kim et al. (1996), the occurrence of OD would be the sum of the means of columns 4-6 divided by 8 , and this yields a probability of 1.00 . This is not as good a fit.

\section{OA-Only Trials}

The a priori probability of an OA-only trial uses the two calculations above, assuming $\mathrm{IM}$ and $\mathrm{OD}$ are uncorrelated, which is essentially true: $p(\mathrm{OA}$-only $)=1-[p(\mathrm{IM})+p(\mathrm{OD})-p(\mathrm{IM})$. $p(\mathrm{OD})]=1-.22-.19+.05=.64$. The empirical probability of an OA-only trial, not considering DMP, was .67. This value is not found in Table 1, because without DMP its value was not relevant to the discussion. Nonetheless, again, it is a reasonable fit. From the Table 2 of $\mathrm{Kim}$ et al. (1996), the predicted value is .00; again, not a good fit.

Thus, Kim et al. (1996) and I agree on the relative occurrence of DMP and IM, but not on OD or OA-only trials. (Kim et al. reported that DMP was a reliable predictor of performance, but did not test for the predictiveness of IM or OD.) What might account for the difference between this analysis and that of Kim et al.? Kim et al. seem to have measured pixel displacements on the screen across pairs of frames. This method suffers from two problems: (a) Not every adjacent pixel pair is separated by the same visual angle and (b) at a given temporal resolution, cross-frame pixel measurements on a raster-scan system suffer spatial aliasing; that is, continuous changes are rounded to the nearest pixel position.

\title{
Appendix B
}

\author{
Conclusions of Kim, Growney, and Turvey (1996)
}

The conclusions of Kim et al. (1996) differ from those presented here. In particular, they concluded that the appropriate information for wayfinding is in optical flow, that expansion is an explanatory variable and a property of optical flow, and that the local sources I have outlined are indistinct and unlikely to account for the data. Let me consider each of these claims in turn.

\section{Optical Flow?}

Although the titles of our articles clash, Kim et al. (1996) and I agree on a fundamental fact: When walking through environments, human beings move their eyes and look at things, executing pursuit fixations on objects around them. Thus, Equation 1 in both Kim et al. and in this Observation are the same. From this equation it is thus easy to see why it is difficult to separate information in retinal flow from that in optical flow. In particular, anything present in optical flow will be present in retinal flow as well. Not everything in retinal flow, however, is present in optical flow unless one happens to be looking in the direction of one's heading. From my perspective, the task of their separation is not as difficult as it might appear. Consider two notions that corroborate use of local motion information in retinal flow.

The first is related to a misattribution of Kim et al. (1996). They found no differences between results for stimuli that simulated linear translation of the observer during pursuit fixation, and those that added vertical and horizontal oscillatory translations and rotations (bounce and sway). These extra motions are signatures of bipedal gait. This nonresult replicates a finding that my colleagues and I have consistently reported (Cutting et al., 1992; Cutting et al., in press; Vishton \& Cutting, 1995). Kim et al. then suggested that the parity of such results cannot then be used as "evidence against the detection of optical flow for translation and as evidence for the use of DMP" (p. 1287). I agree, as did Cutting et al. who, after discussion of their Experiments 1 and 2, stated that "Either decomposition proceeds seamlessly, or it occurs not all" (p. 53). Only later, with their Experiments 8 and 9, did Cutting et al. conclude that information for wayfinding must be in retinal flow. There they exaggerated translational components of bounce and sway, removed the rotational components, and found that performance declined sharply. When the rotational components were added back in, good performance returned. If decomposition and the utility of optical flow were to reign, performance should have been the same in both conditions. Kim et al. have no result or claim that speaks to these results.

Second, and more pertinent to these analyses, if DMP (or DDLO), IM, or OD predicts the perceived direction of heading, then this feat can only be a result of local interaction between the rotation and translation in retinal flow. Global analyses, on which decomposition of rotational and translational flow would depend, do not account for these data as well as the local analyses.

\section{Expansion?}

Kim et al. (1996) took great care to describe what they meant by expansion, considering it to be a property of optical flow, and made much of the difference between the results of the two conditions of Experiment 1, one with expanding stimuli and one without. Cutting (1986, Experiments 9-11) used nonexpanding stimuli and found adequate performance, but also trained observers with feedback to encourage the perception of rigidity. This methodology was chosen because it had been used by Regan and Beverley (1982), whose observers' performance did not improve with feedback; those of Cutting (1986) did, clearly indicating that observers learned to see depth in the displays. Warren et al. (1988) properly criticized the use of feedback, and my colleagues and I have not used it again. An interpretation of the results of the Kim et al. nonexpansion condition, then, is that their observers failed to learn that the stimuli represented rigid environments.

Moreover, there are at least three problems in attributing the other results to expansion. First, by their Equation 1, expansion would be equally a property of retinal flow, and thus could not be used to distinguish theories. Second, even if expansion were a property of optical flow only, half of the stimuli in Experiment 1 and all the stimuli in Experiment 2 were identical in their expansion component, yet performance varied only with the rotational component of Equation 1, generated by changes in the gazemovement angle. Thus, expansion as they define it is uncorrelated with their results. Third, consider how the stimuli were generated. The height and the base of the schematic trees covaried systematically with distance. In the traditional literature these variations are called relative size and height in the visual field, respectively. Both are considered static sources of information (or cues) about 
layout (or depth, see Cutting \& Vishton, 1995, for a discussion). Occlusion, height in the visual field, relative size, and binocular disparities have been shown to contribute to performance on wayfinding tasks (Cutting et al., 1992, Experiment 2; Van den Berg \& Brenner, 1994a, 1994b; Vishton \& Cutting, 1995; Vishton, Nijhawan, \& Cutting, 1994). The results of Kim et al.'s (1996) Experiment 1 thus add to this literature. The way in which such sources aid wayfinding, however, can only be through the establishment of depth relations that are done prior to the use of flow, an idea that Kim et al. eschewed.

\section{Indistinctness of Information?}

DMP (or DDLO), IM, and OD are all conceived of as local information sources. That is, they arise from particular objects in the retinal displacement field. Kim et al. (1996), however, suggested that IM and OD "are poorly suited for a general role in wayfinding" in part because they "tend to co-occur ... in many variants culminating in a retinal flow in which their individual cue values are compromised" (p. 1284). Indeed, these sources co-occur within trials, but only about as often they do not. In fact, out of 896 trials in Experiment 1, only 36 (or 4\%) simultaneously presented proper DMP, IM, and OD information. Out of 112 trials in Experiment 2 , only 5 (or $4 \%$ ) simultaneously presented IM and OD. Thus, the intercorrelations (all $r s<.17$ ) were never large in the stimuli the authors generated. (Kim et al. also included OA in this discussion, but because OA existed on every trial it is not correlated with the results.)

The authors also suggested that "any observation of performance enhanced by IM is probably due to the confound of IM with large gaze angles, at which participants necessarily perform better" (Kim et al., 1996, p. 1286). Indeed, as shown in Figure 1, the area of the terrain in which IM occurs increases with gaze-movement angle. However, Figures 3 and 5 show that when IM is not present, and thus no "confound" present, performance is worse, as the partial correlations attest.

Received August 16, 1995

Revision received November 2, 1995

Accepted November 27, 1995 